\title{
Optimum Centralized Portfolio Construction with Decentralized Portfolio Management
}

\author{
Edwin J. Elton*and Martin J. Gruber*
}

October 8, 2002

* Nomura Professors of Finance, Stern School of Business, New York University. We would like to thank Stephen Brown for his helpful comments on our manuscript. 
Many financial institutions employ outside portfolio managers to manage part or all of their investable assets. These institutions include pension funds, private endowments (e.g., colleges and charities), and private trusts. In 1999, the investment company institute estimated that these institutions managed 5.2 trillion dollars in assets. Most of these institutions employed outside managers to invest these funds. The relevancy of this problem has been widely recognized in the practitioners literature on portfolio $?^{1}$ Furthermore, it is recognized in the prudent man law that spells out the responsibilities of the centralized decision maker delegating management responsibility. ${ }^{2}$ For example the New York State law in estate power and trust states. Pension funds are the largest and most likely organizations to employ several outside managers, each of whom manages a part of the overall portfolio. In this paper we will use the pension fund manager as the prototype of the centralized decision-maker trying to optimally manage a set of decentralized portfolio managers but the analysts is general.

If the centralized decision-maker (CDM) is a mean variance maximizer, the CDM could construct a portfolio using standard portfolio theory and estimates of mean return, variances, and covariances between the portfolios constructed by a group of decentralized managers. However, this overall portfolio is unlikely to be optimum since the individually managed portfolios themselves were constructed without taking into account the portfolios of the other managers. The purpose of this article is to set up a structure that leads to the optimum portfolio from the viewpoint of the CDM when there are multiple managers and their portfolios are constructed without reference to each other.

\footnotetext{
${ }^{1}$ See, for example, Di Bartolomer (1999), Grinald and Kahn (1995), Farrell (1976), and Rosenberry (1977).

${ }^{2}$ For a full discussion of modern portfolio theory and the prudent man rule see Elton and Gruber in Longstaff ( ).
} 
This paper can be viewed as a contribution to the extensive literature in Financial Economics developing conditions under which a CDM will never make a worse decision than decentralized managers providing the information is used optimally. ${ }^{3}$ This literature assumes the decentralized managers are willing to provide all information to each other or to a centralized manager. This case has been examined for the pension fund problem by Rosenberg (1977) and D. Bartolomeo (1999). For example, Rosenberg (1977) demonstrated that with full information, the decentralized portfolio managers will not make better decisions than that of the centralized manager. This paper examines a special case of this more general literature: the case of a centralized portfolio manager employing a set of individual portfolio managers each of whom constructs his or her own portfolio without communicating with other managers. Several authors have asserted that this problem is too difficult to solve (see Rosenberg (1977) and D. Bartolomeo (1999)). They argue the only solutions are for each outside manager to either turn over all of their estimates for individual security characteristics to a centralized manager or to supply all purchases and sales to the centralized managers. In the latter case models are presented that allow the central manager to approximate from this information, the individual managers' forecasts. What makes this a special case is the realistic assumption that a decentralized manager is only willing to share some information with the centralized manager and none with other managers. In this article, we solve for sets of conditions under which the centralized manager can make optimum decisions despite partial information through the use of guidelines for the decentralized managers. While this can be viewed as an extension of the previous literature on centralized versus decentralized

\footnotetext{
${ }^{3}$ See, for example, Radner (1962), Marshak and Radner (1972), and Ohlson (1975 and 1979).
} 
decision making it is of at least equal importance because it offers a solution to a problem which is at the heart of investment allocation today.

In the first section we will present a more detailed discussion of the problem. We will then solve the problem for one active manager and multiple passive portfolios. The model is then generalized to multiple active managers. Next, we present solutions under a simplified structure of the return-generating process. Finally, we discuss the complications when short sales are not allowed.

\section{Background}

In this section we discuss some background material on the pension investment problem and review the relevant literature. The same considerations hold for private endowments and trusts. Most pension plans are managed by a centralized decision maker at a firm. Most firms have one person who is principally in charge, although the ultimate responsibility rests with a committee, usually the board. This CDM normally employs outside portfolio managers to construct active portfolios. Index funds are generic products and we will assume the centralized decision maker can potentially select one or more of these. The centralized decision maker's task is fourfold: 1) decide how much to invest in each portfolio, 2) give the outside managers instructions that will result in their making optimum security allocations from the point of view of the overall plan, 3) design incentive systems so that the managers will behave optimally ${ }^{4}$, and 4) evaluate and select the portfolio managers. In this paper we deal only with the first two of these problems although our solutions have major implications for the third and fourth problems. Throughout the paper, we assume that the portfolio managers will not provide the

\footnotetext{
${ }^{4}$ No one has addressed the multi-period incentive problem outlined here. However, there are a number of related articles. See for example, Dyvig, Farnsworth and Carpenter (2001), Kihlstrom (1988), Stoughton (1993), and Zender (1988).
} 
centralized decision maker with their return forecasts for individual securities, but will provide aggregate information about the portfolios they hold.

Aspects of this problem have previously been addressed by Treynor and Black (1973) and in more detail by Sharpe (1981). The Treynor Black article discussed the active passive split when the CAPM described the returns on the passive portfolio, short sales are allowed and the single-index model describes the return generating process. The clear antecedent to this article is Sharpe's (1981) Presidential address. Sharpe develops, with one active and one passive manager, the instructions for the active manager that will result in the active manager producing a globally optimal portfolio for a particular utility function. He assumes short sales are allowed and the variance covariance matrix is agreed on by all parties. He also solves for the instructions to be given to the managers that results in a global optimal for the case of two managers following exactly the same set of securities where the centralized decision maker believes the best forecast of a securities alpha is a weighted average of the two managers alphas and where these weights add to one. In solving this problem he maintains the assumption of short sales allowed and agreement on the variance covariance matrix. Sharpe could not obtain an exact solution for the case of managers following non-overlapping securities. Our analysis extends Sharpe in that we generalize to $N$ managers, have no requirement that each manager holds the same securities, and, by employing a multifactor model, can arrive at simple rules for forming myopic optimum portfolios, understanding the weight placed on each security in these portfolios, and the amount to allocate to each active and passive portfolio. We also extend the analysis to the case 
where short sales are not allowed and show conditions under which optimal decentralized management is possible and when it is not.

\section{Separation with a single active and multiple passive managers}

In this section of the paper we will assume that a centralized decision maker (CDM) exists who hires a single active manager. We will shortly expand the case to several active managers. We will assume the following: 1) the CDM is a mean variance decision maker, 2) the CDM believes a multi-index model describes the return structure for securities and all indexes in the multi-index model are tradable.

The second point requires some clarification. The CDM believes that returns can be described as being generated by a set of indexes (not necessarily orthogonal) that the CDM can take positions in as passive portfolios $^{5}$. For example, this is consistent with a belief that the return on securities is a function of the market return, the return on a portfolio of small stocks, and/or the return on a portfolio of value or growth stocks. The CDM wishes to consider these sources of risk in making the optimum mean variance decision. For expositional reasons we will analyze the CDM's problem with a two index model though the solution easily generalizes to any number of indexes.

\section{A. The CDM's problem}

We start by examining the optimum decision the CDM would make if the CDM had all the information that is available to the active managers. As mentioned earlier, we believe the CDM would not be able to obtain risk adjusted return forecasts for individual securities from the active manager, but for the moment we examine the optimum decision

\footnotetext{
${ }^{5}$ Index funds, many of them exchange traded, exist for almost any index a manager might want to use in a return generating process.
} 
as if the CDM has such information. We will also assume that the CDM does not have perfect faith in the return forecasts of the active manager. This implies that the CDM will take positions in the passive portfolios for two reasons, to obtain diversification across securities so that the aggregate portfolio is mean variance efficient, and to eliminate some of the lack of reliability in the analyst's estimates.

In order to specify the return generating process, define

1. $R_{i}$ is the return on stock $i$

2. $R_{F}$ is the risk free rate of interest

3. $R_{A}, R_{B}$ is the return on index $A$ and index $B$ respectively

4. $\quad \beta_{i A}, \beta_{i B}$ is the sensitivity of stock $i$ to indexes $A$ and $B$

5. $\sigma_{A}^{2}, \sigma_{B}^{2}$ is the variance of the return on indexes $A$ and $B$

6. $\sigma_{e i}^{2}$ is the residual risk of stock $i$ from the two-index model

7. $\quad \alpha_{i}$ is the risk adjusted return on security $i$

8. $\quad e_{i}$ is the residual return for security $i$

9. The superscript $D$ designates that the decision is from the point of view of the CDM.

Then the return generating process is

$R_{i}-R_{F}=\alpha_{i}+\beta_{i A}\left(R_{A}-R_{F}\right)+\beta_{i B}\left(R_{B}-R_{F}\right)+e_{i}$

Assume that the CDM had access to the excess return forecasts $\left(\alpha_{i}\right)$ of the active manager. Furthermore, assume the CDM believes that the best estimate of risk-adjusted excess return is an average of the analysts' forecasts and the value that would occur in 
equilibrium namely zero. Thus, we define the excess risk adjusted return that the CDM would use as $\alpha_{i}^{D}=W \alpha_{i}$ where $W$ is set by the CDM between 0 and $1^{6}$.

To solve this problem, assuming short sales, the CDM can use the standard first order conditions. The investments that can be selected are the $N$ individual securities and the two indexes. The first order condition for security and index $i$ is

$$
\bar{R}_{i}-R_{F}=Z_{i}^{D} \sigma_{i}^{2}+\sum_{\substack{j=1 \\ j \neq i}}^{N+2} Z_{j}^{D} \sigma_{i j} \text { for } i=1, \ldots, N+2
$$

Where

1. $\quad N$ is the number of securities entering into the decision making process

2. Security $N+1$ and $N+2$ are indexes which we henceforth designate as $A$ and $B$.

3. $Z_{i}^{D}$ is a number proportional to the optimal weight which the CDM would place in security $i$

If the return generating process described in equation (1) is an accurate description of returns and we recognize that the indexes need not be orthogonal, then we can define the variance and covariance between individual securities as

$$
\begin{array}{lll}
\sigma_{i}^{2}=\beta_{i A}^{2} \sigma_{A}^{2}+\beta_{i B}^{2} \sigma_{B}^{2}+2 \beta_{i A} \beta_{i B} \sigma_{A B}+\sigma_{e i}^{2} & \text { for } i=1, \ldots, N & \\
\sigma_{i j}=\beta_{i A} \beta_{j A} \sigma_{A}^{2}+\beta_{i B} \beta_{j B} \sigma_{B}^{2}+\beta_{i A} \beta_{j B} \sigma_{A B}+\beta_{i B} \beta_{j A} \sigma_{A B} & \text { for } i=1 \ldots N & j=1, \ldots N \\
& i \neq j
\end{array}
$$

\footnotetext{
${ }^{6}$ While the optimum way to set $W$ is beyond the scope of this paper, there have been a number of excellent articles published in the past few years explaining optimum ways of changing alpha for estimation risk and bias. See Bawa, Brown, and Kleen (1979) for the fundamental application of Baysian analysis and Baks, Metrick, and Wachter (2001) and Pastor and Stambaugh (2001) for recent applications of Baysian analysis to estimating the inputs for optimal portfolio allocation.
} 
For the $N+1$ and $N+2$ securities (the indexes), a simpler form exists. For example, for index $A$ the variance is $\sigma_{A}^{2}$ and the covariance with index $B$ is $\sigma_{A B}$ and the covariance with individual securities is

$\sigma_{i A}=\beta_{i A} \sigma_{A}^{2}+\beta_{i B} \sigma_{A B}$

Employing these relationships with the first order condition (2), we get for security $i$

$W \alpha_{i}+\beta_{i A}\left(\bar{R}_{A}-R_{F}\right)+\beta_{i B}\left(\bar{R}_{B}-R_{F}\right)=$

$Z_{i}^{D} \beta_{i A}^{2} \sigma_{A}^{2}+Z_{i}^{D} \beta_{i B}^{2} \sigma_{B}^{2}+2 Z_{i}^{D} \beta_{i A} \beta_{i B} \sigma_{A B}+Z_{i}^{D} \sigma_{e i}^{2}+$

$\beta_{i A} \sum_{\substack{j=1 \\ j \neq i}}^{N} Z_{j}^{D} \beta_{j A} \sigma_{A}^{2}+\beta_{i B} \sum_{\substack{j=1 \\ j \neq i}}^{N} Z_{j}^{D} \beta_{j B} \sigma_{B}^{2}+\beta_{i A} \sum_{\substack{j=1 \\ j \neq i}} Z_{j}^{D} \beta_{j B} \sigma_{A B}+$

$\beta_{i B} \sum_{\substack{j=1 \\ j \neq i}}^{N} Z_{j}^{D} \beta_{j A} \sigma_{A B}+Z_{A}^{D} \beta_{i A} \sigma_{A}^{2}+Z_{A}^{D} \beta_{i B} \sigma_{A B}+Z_{B}^{D} \beta_{i B} \sigma_{B}^{2}+Z_{B}^{D} \beta_{i A} \sigma_{A B}$

and for the indexes

$$
\begin{aligned}
& \bar{R}_{A}-R_{F}=Z_{A}^{D} \sigma_{A}^{2}+\sum_{j=1}^{N} Z_{j}^{D} \beta_{j A} \sigma_{A}^{2}+\sum_{j=1}^{N} Z_{j}^{D} \beta_{j B} \sigma_{A B}+Z_{B}^{D} \sigma_{A B} \\
& \bar{R}_{B}-R_{F}=Z_{B}^{D} \sigma_{B}^{2}+\sum_{j=1}^{N} Z_{j}^{D} \beta_{j B} \sigma_{B}^{2}+\sum_{j=1}^{N} Z_{j}^{D} \beta_{j A} \sigma_{A B}+Z_{A}^{D} \sigma_{A B}
\end{aligned}
$$

Substituting equations (4) and (5) into equation (3) and simplifying, we get ${ }^{7}$

$$
Z_{i}^{D}=\frac{\alpha_{i}^{D}}{\sigma_{e i}^{2}}=\frac{W \alpha_{i}}{\sigma_{e i}^{2}}
$$

To solve for the optimum amount in security $i$ we consider the active portfolio denoted by $P$ as a separate portfolio and look at the optimum composition of this portfolio before we allocate across all three portfolios. We can treat the design of $P$ as a separate portfolio because from equation (6), $Z_{i}^{D}$ is not a function of $Z_{A}$ or $Z_{B}$.

\footnotetext{
${ }^{7}$ A similar expression but in a different context can be found in Elton and Gruber and Padberg (1979).
} 
The fraction to invest in any stock, $X_{i p}$, in the active portfolio can be determined by recognizing that ${ }^{8} X_{i p}=\frac{Z_{i}^{D}}{\Sigma Z_{i}^{D}}$. Therefore, recognizing that the amount to invest in an stock $i$ in the optimal active portfolio from the viewpoint of the CDM is

$$
X_{i p}=\frac{\alpha_{i}^{D} / \sigma_{e i}^{2}}{\sum_{j=1}^{N} \alpha_{j}^{D} / \sigma_{e j}^{2}}=\frac{\frac{\alpha_{i}}{\sigma_{e i}^{2}}}{\sum_{j=1}^{N} \frac{\alpha_{t j}}{\sigma_{e j}}}
$$

Once portfolio $P$ is determined simple procedures exist for allocating funds between the active and passive portfolios. These are presented in Section $\mathrm{C}$ below.

\section{B. Optimum active portfolio}

The CDM can ensure that the active manager will hold the optimal active portfolio from the point of view of the CDM simply by instructing the active manager to compute by $\alpha_{i} / \sigma_{e i}^{2}$ for each stock and to hold them in that proportion. ${ }^{9}$ This simple instruction ensures that the active manager will turn over to the CDM the same active portfolio that the CDM would hold if all the security estimates were supplied directly to the CDM. Optimization for the active portfolio is reached without the active manager giving up private information.

Of course the CDM still has the problem of deciding what fraction of funds to place in the active portfolio and each of the passive portfolios.

\footnotetext{
${ }^{8}$ See Elton, Gruber, and Padburg (1976) for a full exposition or Lintner (1965) for the original proof. ${ }^{9}$. If the decentralized manager were simply told to form the optimum active portfolio assuming that he could hold the passive portfolio, he would get the same result as following the direction from the central manager. Although this ranking device was derived in the prior section using two indexes it is easy to show that the same ranking devise holds if there are $N$ indexes
} 


\section{Solving the aggregate allocation problem}

Denote the characteristics of the active portfolio by the subscript $P$. Then from the viewpoint of the $\mathrm{CDM}$, ignoring for the moment any difficulty of getting information, the problem can be formulated and solved using the following first order conditions ${ }^{10}$.

$$
\begin{aligned}
& \alpha_{P}^{D}+\beta_{P A}\left(\bar{R}_{A}-R_{F}\right)+\beta_{P B}\left(\bar{R}_{B}-R_{F}\right)= \\
& Z_{P}^{D}\left(\beta_{P A}^{2} \sigma_{A}^{2}+\beta_{P B}^{2} \sigma_{B}^{2}+2 \beta_{P A} \beta_{P B} \sigma_{A B}+\sigma_{e P}^{2}\right)+Z_{A}^{D} \beta_{P A} \sigma_{A}^{2}+Z_{B}^{D} \beta_{P B} \sigma_{B}^{2} \\
& \bar{R}_{A}-R_{F}=Z_{P}^{D}\left(\beta_{P A} \sigma_{A}^{2}+\beta_{P B} \sigma_{A B}\right)+Z_{A}^{D} \sigma_{A}^{2}+Z_{B}^{D} \sigma_{A B} \\
& \bar{R}_{B}-R_{F}=Z_{P}^{D}\left(\beta_{P B} \sigma_{B}^{2}+\beta_{P A} \sigma_{A B}\right)+Z_{B}^{D} \sigma_{B}^{2}+Z_{A}^{D} \sigma_{A B}
\end{aligned}
$$

These are standard first order conditions. Since everything but the $Z$ 's are known, the equations can be solved explicitly for the optimal fraction of funds in each portfolio.

To do this we utilize a relationship we derive later. As shown in equation (13),

$\Sigma \frac{\alpha_{j}}{\sigma_{e j}^{2}}=\frac{\alpha_{P}}{\sigma_{e P}^{2}}$. Using this expression, the solution is

$Z_{A}^{D}=\frac{\left(\bar{R}_{A}-R_{F}\right)}{\sigma_{A}^{2}\left(1-\rho^{2}\right)}-\frac{\left(\bar{R}_{B}-R_{F}\right) \rho}{\sigma_{A} \sigma_{B}\left(1-\rho^{2}\right)}-\beta_{P A} W \frac{\alpha_{P}}{\sigma_{e P}^{2}}$

$Z_{B}=\frac{\bar{R}_{B}-R_{F}}{\sigma_{B}^{2}\left(1-\rho^{2}\right)}-\frac{\left(\bar{R}_{A}-R_{F}\right)}{\sigma_{A} \sigma_{B}\left(1-\rho^{2}\right)}-\beta_{P B} W \frac{\alpha_{P}}{\sigma_{e P}^{2}}$

$Z_{P}=W \frac{\alpha_{P}}{\sigma_{e P}^{2}}$

Where $\rho$ is the correlation between passive portfolio A and $\mathrm{B}$. These three equations along with the expression normalizing the portfolio weights to add to one which is

\footnotetext{
${ }^{10}$ The extension to more than two indexes is straightforward. One new equation would be added for each index, one $Z$ for each new index would be added to each equation and the variance and covariance terms would be modified to account for the additional indexes.
} 


$$
X_{k}=\frac{Z_{k}}{\Sigma_{l} Z_{l}} \quad l=A, B, \text { and } P
$$

give us the closed form solution for the optimal weight to place in the active and each passive portfolio.

The optimal weights depend on the fundamental characteristics of each of the three portfolios in a way that makes intuitive sense. For each of the passive index funds, the higher the excess return on the fund relative to its variance, the larger the allocation of funds to that portfolio. Similarly, for the active portfolio, the larger the risk adjusted return for that portfolio relative to its unsystematic risk, the greater the funds placed in it. The correlation coefficient between the indexes also has a large effect on the relative investment in each of the passive portfolios. The impact of the correlation coefficient on allocation depends on the ratio of the excess return to standard deviation of index A to that of index B as well as the size of the correlation coefficient itself. In order to determine the split across portfolios, the CDM needs to request the active manager's estimate of the alpha for the active portfolio, the residual risk of the active portfolio and the active portfolio sensitivities to the two indexes. These are the types of estimates the active manager should be willing to supply since they are aggregate portfolio values rather than individual security values. ${ }^{11}$ The CDM needs to estimate the expected return above the riskless rate and risk on the passive funds, the covariance between the passive

\footnotetext{
${ }^{11}$ As stated earlier, we are assuming that the CDM and the active manager are employing identical estimates of the $\beta$ 's and residual risks but not return characteristics of each security. This could come about naturally if the risk parameters were estimated from the same commercial service (e.g., BARA or Wilshire). The CDM could either specify that decentralized managers use a particular commercial service or directly supply the risk parameters for the assumption of our model to hold. The need for a common return generating process might particularly explain the specification of benchmarks in contracts with managers.
} 
funds, and the amount of weight $(W)$ to put on the active manager's estimates. If futures are available on the indexes, the aggregate portfolio problem is simplified.

\section{The Aggregate Portfolio Problem with Futures}

If futures are available on the indexes, then sensitivities to the indexes can be adjusted without affecting the amount invested in the active portfolio. The expected return and risk on the portfolio of the $\mathrm{CDM}$ is,

$$
\begin{aligned}
& \bar{R}_{C}-R_{F}=X_{P} W \alpha_{P}+\beta_{A}\left(\bar{R}_{A}-R_{F}\right)+\beta_{B}\left(\bar{R}_{B}-R_{F}\right) \\
& \sigma_{C}^{2}=X_{P} \sigma_{e P}^{2}+\beta_{A}^{2} \sigma_{A}^{2}+\beta_{B}^{2} \sigma_{B}^{2}+2 \beta_{A} \beta_{B} \sigma_{A B}
\end{aligned}
$$

where $C$ is the overall portfolio held by the CDM. The choice variables for the CDM are how much to put in the active portfolio, how much to place in the riskless asset and the level of sensitivity of the overall portfolio to each of the factors. Taking derivatives of $\theta=\frac{\bar{R}_{C}-R_{F}}{\sigma_{C}}$ with respect to $X_{P}, \beta_{A}$ and $\beta_{B}$ respectively results in the following first order conditions

$$
\begin{aligned}
& \frac{\bar{R}_{C}-R_{F}}{\sigma_{C}^{2}} X_{P}=\frac{\sigma_{P}^{D}}{\sigma_{e P}^{2}}=W \frac{\alpha_{P}}{\sigma_{e P}^{2}} \\
& \frac{\bar{R}_{C}-R_{F}}{\sigma_{C}^{2}}\left(\beta_{A} \sigma_{A}^{2}+2 \beta_{B} \sigma_{A B}\right)=\bar{R}_{A}-R_{F} \\
& \frac{\bar{R}_{C}-R_{F}}{\sigma_{C}^{2}}\left(\beta_{B} \sigma_{B}^{2}+2 \beta_{A} \sigma_{A B}\right)=\bar{R}_{B}-R_{F} .
\end{aligned}
$$

The efficient frontier is the line connecting the riskless asset with the optimal risky portfolio. If we can determine one point on the line, we can trace out the full efficient 
frontier. Varying $X_{P}$ traces out the line. Thus, with no loss of generality we can solve for the portfolio with $X_{P}=1$. Setting $X_{P}$ equal to one we get that the optimum betas are

$$
\begin{aligned}
& \beta_{A}=\frac{\left[\frac{\left(\bar{R}_{A}-R_{F}\right)}{\sigma_{A}^{2}}-\frac{\left(\bar{R}_{B}-R_{F}\right)}{\sigma_{A} \sigma_{B}} \rho_{A B}\right] \frac{\sigma_{e P}^{2}}{W \alpha_{P}}}{\left(1-\rho_{A B}^{2}\right)} \\
& \beta_{B}=\frac{\left[\frac{\bar{R}_{B}-R_{F}}{\sigma_{B}^{2}}-\frac{\left(\bar{R}_{A}-R_{F}\right)}{\sigma_{A} \sigma_{B}} \rho_{A B}\right] \frac{\sigma_{e P}^{2}}{W \alpha_{P}}}{\left(1-\rho_{A B}^{2}\right)}
\end{aligned}
$$

where $\rho_{A B}$ is the correlation between the two indexes.

The easiest way to interpret the results is to consider the case $\rho_{A B}$ equal to zero. With this assumption, $\beta_{A}$ is equal to the excess return to risk of index $A$ divided by the ratio of the risk adjusted return to the residual risk of the active portfolio. When the indexes are correlated, this ratio is modified to take account the correlation between the indexes.

We have now presented a set of conditions under which a centralized decisionmaker can optimize portfolio composition while employing one active manager. The next problem to solve is the case where the CDM employs several active managers.

\section{Multiple active managers}

The analysis generalizes to multiple active managers whether these managers follow some or all securities in common or follow independent sections of the market. ${ }^{12}$ For

\footnotetext{
${ }^{12}$ Sharpe (1981) did not reach an explicit solution in the case where only some securities were in common across active managers.
} 
simplicity we will solve for the case of two active managers, but the analysis easily generalizes. Assume that the CDM has all the information produced by each manager but different confidence in the forecasts of each manager. Furthermore, the CDM believes that all the managers $\alpha$ estimates are too extreme but that the appropriate estimate is some combination of them. ${ }^{13}$ If we designate the weight the CDM puts on the estimate prepared by manager 1 as $W_{1}$ and manager 2 as $W_{2}$. Then $\alpha_{i}^{D}=W_{1} \alpha_{i 1}+W_{2} \alpha_{i 2}$. Once again it is necessary for the CDM to supply estimates of betas and residual variances to all active managers either directly or by specifying that they use a common service such as BARRA. Since $\sigma_{e i}^{2}$ is supplied by the CDM to all managers, it is common and

$$
\frac{\alpha_{i}^{D}}{\sigma_{e i}^{2}}=W_{1} \frac{\alpha_{i 1}}{\sigma_{e i}^{2}}+W_{2} \frac{\alpha_{i 2}}{\sigma_{e i}^{2}}
$$

Earlier we showed that $\frac{\alpha_{i}^{D}}{\alpha_{e i}^{2}}$ was proportioned to the optimum amount that the CDM wished to place in security $i$ if all alphas were supplied to the CDM. The issue we address in this section is the instructions to give to the individual managers and the correct proportions to invest in each active portfolio so that the CDM, by combining the portfolios of the active managers, ends up with a fraction in the active portfolio proportional to $\alpha_{i}^{D} / \sigma_{e i}^{2}$ for each security $i$

Summing both sides of equation (9) across all securities

\footnotetext{
${ }^{13}$ Implicit in what follows is if only one manager follows a security, the CDM assumes the best estimate of the second manager's alpha if he/she followed it would be zero.
} 
$\sum_{i} \frac{\alpha_{i}^{D}}{\sigma_{e i}^{2}}=W_{1} \sum_{i} \frac{\alpha_{i 1}}{\sigma_{e i}^{2}}+W_{2} \sum_{i} \frac{\alpha_{i 2}}{\sigma_{e i}^{2}}$

If the CDM instructs each manager to compute $\alpha_{i} / \sigma_{e i}^{2}$ for each security and to place a fraction of money in each security proportional to this ratio we can define the fraction any manager (e.g., manager 1) places in any security as

$$
X_{i 1}=\frac{\frac{\alpha_{i 1}}{\sigma_{e i}^{2}}}{\sum_{j=1}^{N} \frac{\alpha j}{\sigma_{e j}^{2}}}
$$

We will now show this instruction results in an overall optimum. However, before we do so, we need to derive some of the attributes of the portfolio which manager 1 (or any manager) will hold. The risk-adjusted excess return on the portfolio held by manager 1 is

$$
\alpha_{P 1}=\sum_{j} X_{j 1} \alpha_{j 1}=\frac{\sum_{j}\left[\left(\frac{\alpha_{j 1}}{\sigma_{e j}^{2}}\right) \alpha_{j 1}\right]}{\sum_{j} \frac{\alpha_{j 1}}{\sigma_{e j}^{2}}}=\frac{\sum\left[\left(\frac{\alpha_{j 1}^{2}}{\sigma_{e j}^{2}}\right)\right]}{\sum_{j} \frac{\alpha_{j 1}^{2}}{\sigma_{e j}^{2}}}
$$

and the residual risk of this active portfolio is 


$$
\sigma_{e P 1}^{2}=\Sigma_{j} X_{j}^{2} \sigma_{e j}^{2}=\frac{\Sigma_{j}\left[\left(\frac{\alpha_{j 1}}{\sigma_{e j}^{2}}\right)^{2} \sigma_{e j}^{2}\right]}{\left[\Sigma_{j}\left(\frac{\alpha j}{\sigma_{e j}^{2}}\right)\right]^{2}}=\frac{\left[\left(\begin{array}{c}
\alpha_{j}^{2} \\
\sigma_{e j}^{2}
\end{array}\right)\right]}{\left[j\left(\frac{\alpha_{j 1}^{2}}{\sigma_{e j}^{2}}\right)\right]^{2}}
$$

Taking the ratio of (11) and (12) yields

$$
\frac{\alpha_{P 1}}{\sigma_{e P 1}^{2}}=\sum_{j} \frac{\alpha_{j 1}}{\sigma_{e j}^{2}}
$$

Furthermore, $\beta_{P 1 K}$ where the subscript $K$ is a counter, indication either index $A$ or index $B$ equals

$$
\beta_{P 1 K}=\sum_{j} X_{j 1} \beta_{j k}=\frac{\sum_{j}\left(\frac{\alpha}{\sigma_{j 1}^{2}} \beta_{j K}\right)}{\sum_{j} \frac{\alpha}{\sigma_{e j}^{2}}}
$$

Rearranging and substituting equation (13) yields

$$
\sum_{j} \frac{\alpha_{j 1} \beta_{j K}}{\sigma_{e j}^{2}}=\beta_{P 1 K} \frac{\alpha_{P 1}}{\sigma_{e P}^{2}}
$$

Having developed these expressions, we can now show that there exists an allocation across the active portfolios along with the instruction to the individual managers to hold 
stocks in proportion $\alpha_{i} \sigma_{e i}^{2}$, which results in an overall optimum to the CDM.

Substituting equation (13) into (10) yields

$\frac{\alpha_{P}^{D}}{\sigma_{e P D}^{2}}=W_{1} \frac{\alpha_{P 1}}{\sigma_{e P 1}^{2}}+W_{2} \frac{\alpha_{P 2}}{\sigma_{e P 2}^{2}}$

Recall that the individual portfolio manager has been instructed to form a

portfolio by holding securities proportional to the ratio of excess return to residual risk.

Recognizing this instruction and using equation (13) to simplify the denominator

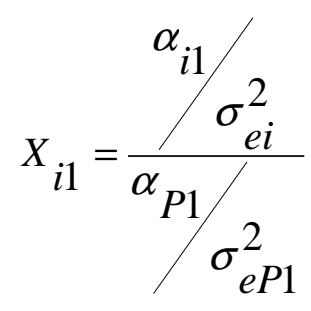

Dividing both sides of equation (9) by $\frac{\alpha_{P}^{D}}{\sigma_{e P D}^{2}}$, the correct amount in security $i$ in the active portfolios from the point of view of the CDM is

$X_{i}^{D}=\frac{\alpha_{i}^{D} \int_{P i}^{2}}{\alpha_{P}^{D} \sigma_{e P D}^{2}}=W_{1}\left[\frac{\frac{\alpha_{P 1}}{\sigma_{e P 1}^{2}}}{\frac{\alpha_{P D}}{\sigma_{e P D}^{2}}}\right]\left[\frac{\frac{\alpha_{i 1}}{\sigma_{e 1}^{2}}}{\frac{\alpha_{P 1}}{\sigma_{e P 1}^{2}}}\right]+W_{2}\left[\frac{\frac{\alpha_{P 2}}{\sigma_{e P 2}^{2}}}{\frac{\alpha_{P D}}{\sigma_{e P D}^{2}}}\right]\left[\frac{\frac{\alpha_{i 2}}{\sigma_{e 2}^{2}}}{\frac{\alpha_{P 2}}{\sigma_{e P 2}^{2}}}\right]$

$=\left[W_{1} \frac{\frac{\alpha_{P 1}}{\sigma_{e P 1}^{2}}}{\frac{\alpha_{P D}}{\sigma_{e P D}^{2}}}\right] X_{i 1}+\left[W_{2} \frac{\frac{\alpha_{P 2}}{\sigma_{e P 2}^{2}}}{\frac{\alpha_{P D}}{\sigma_{e P D}^{2}}}\right] X_{i 2}$ 
Where the terms in brackets represent the proportion of the active portfolio to invest with manager 1 and manager 2 , respectively. Since $\frac{\alpha_{P}^{D}}{\sigma_{e P D}^{2}}$ can be computed from equation (16), if the CDM obtains $\alpha_{P 1}$ and $\sigma_{e P 1}^{2}$ from manager 1 , and $\alpha_{P 2}$ and $\sigma_{e P 2}^{2}$ from manager 2 , he or she can determine optimum proportions among active managers.

In addition, since the CDM knows the characteristics of the aggregate active portfolio, the CDM can act in determining the split between the active and passive portfolios as if there is a single portfolio. Thus, the allocation between the active portfolio and the two passive portfolios can be determined using the equations in Section II C.

\section{Orthogonal Indexes}

Up to this point we have assumed that the indexes are not orthogonal. The advantage of this is that it allows the passive portfolios to be portfolios that exist in the market such as small stocks, the S\&P Index, growth stocks, etc. However, if we are willing to assume orthogonal indexes the allocation across active and passive managers is simplified. With orthogonal indexes, the covariance among indexes is zero, and there exists a simple formula for the amount to invest in the passive index. For passive index A equation (4) becomes

$$
\bar{R}_{A}-R_{F}=\Sigma_{j} Z_{j}^{D} \beta_{j A} \sigma_{A}^{2}+Z_{A}^{D} \sigma_{A}^{2}
$$

Solving for $Z_{A}^{D}$ 
$Z_{A}^{D}=\frac{\bar{R}_{A}-R_{F}}{\sigma_{A}^{2}}-\sum_{j=1}^{N} Z_{j}^{D} \beta_{j A}$

Substituting for $Z_{j}^{D}$ from equation (6) yields

$Z_{A}^{D}=\frac{\bar{R}_{A}-R_{F}}{\sigma_{A}^{2}}-\sum_{j=1}^{N} \frac{\alpha_{j}^{D}}{\sigma_{e j}^{2}} \beta_{j A}$

Expressing $\frac{\alpha_{j}^{D}}{\sigma_{e i}^{2}}$ in terms of the two active portfolios

$Z_{A}^{D}=\frac{\bar{R}_{A}-R_{F}}{\sigma_{A}^{2}}-W_{1} \sum_{j=1}^{N} \frac{\alpha_{j 1}}{\sigma_{e j}^{2}} \beta_{j A}-W_{2} \underset{j=1}{\sum} \frac{\alpha_{j 2}}{\sigma_{e j}^{2}} \beta_{j A}$

Finally, using equation (15):

$Z_{A}^{D}=\frac{\bar{R}_{A}-R_{F}}{\sigma_{A}^{2}}-\left[W_{1} \frac{\alpha_{P 1}}{\sigma_{e P 1}^{2}} \beta_{P 1 A}+W_{2} \frac{\alpha_{P 2}}{\sigma_{e P 2}^{2}} \beta_{P 2 A}\right]$

Thus, the centralized decision maker can determine the total $Z$ and the split between each of the passive portfolios and each of the active portfolios using a simple formula if all managers provide their estimates of $\alpha_{P}, \sigma_{e P}^{2}$ and $\beta$ on each index, and the centralized decision maker estimates the $W$ 's and excess return and risk on the index. The active managers also need to have common risk measures, $\beta_{i}$ 's and $\sigma_{e i}^{2}$ for all securities under consideration. In the case of orthogonal indexes, characteristics of indexes other than the 
one being analyzed do not impact the $Z$ associated with any index. Thus, the equation applies to any number of indexes ${ }^{14}$.

\section{Short sales not allowed}

Let's start with the case of a single active manager where short sales of the indexes are allowed but short sales of securities are not. This case is realistic for some centralized decision makers. Futures exchange traded funds or future replications with options exist for many indexes. In this case a CDM can effectively short sell indexes. To determine the optimum when securities cannot be short sold, we need to use Kuhn, Tucker conditions. This simply involves adding the dual variables $M_{i}$ 's (one for each security) to equation (3) the first order conditions for each security when short sales are allowed. The solution to the portfolio problem makes use of the complimentary conditions that the product of the dual and the primal must be zero $\left(X_{i} M_{i}=0\right.$ for all $\left.i\right)$ and that $M_{i}$ and $X_{i}$ must be equal to or greater than zero for all $i$. Since there are no duals on the first order conditions for indexes, equations (4) and (5) are unchanged. Equation (6) holds with the addition of the dual for the security ${ }^{15}$. Adding the dual, equation (6) becomes $Z_{i}^{D}=\frac{W \alpha_{i}}{\sigma_{e i}^{2}}+M_{i}^{*}$.

If $\alpha_{i}$ is positive, $Z_{i}^{D}$ must be positive since $M_{i}^{*}$ cannot be negative. If $Z_{i}^{D}$ is positive from the complementary correlation, $M_{i}^{*}$ must be zero. If $\alpha_{i}$ is negative then $M_{i}^{*}$ must be positive so that $Z_{i}^{D}$ is not negative and from the complementary condition

${ }^{15} M_{i}^{*}$ is a transformation of the $M_{i}$ added to each equation, but has the same sign as $M_{i}$. 
$Z_{i}^{D}$ must be zero. Thus, all $Z_{i}^{D}$ either equal $\frac{W \alpha_{i}}{\sigma_{e i}^{2}}$ or zero. The optimum portfolio for the CDM is obtained by having the manager invest in all securities for which $\alpha_{i}>0$ and as before in proportion to $\frac{\alpha_{i}}{\sigma_{e i}^{2}}$. The equations in section II C, then define the optimal split between the active and passive portfolios.

If there are multiple active managers, the condition under which an optimal solution can be reached are more restrictive. To understand the problem, consider the case where manager 1 forecasts $\alpha_{i 1}>0$ and manager 2 forecasts $\alpha_{i 2}<0$ where the absolute value of $\alpha_{i 2}$ is greater than $\alpha_{i 1}$ and the CDM puts equal weights on the estimates of each manager. In this case the CDM would want to hold zero in security $i$. However, manager 1 will hold positive proportions and without short sales, manager 2 will hold zero rather than short sell. No combination will provide an optimum to the CDM.

The only exception to this scenario is the case where the centralized manager wishes to place no weight on a forecast of a negative alpha. This implies that the CDM believes the managers have no ability to forecast below normal returns but have some ability on the upside. In the case where $\alpha_{i 1}>0$ and $\alpha_{i 2}<0$, the CDM would want to use $\alpha_{i}^{D}=W_{1} \alpha_{i 1}$ and, providing all passive portfolios are held long or short sales of passive portfolios are allowed, the analysis outlined above goes through with each active manager not allowed to have short sales. 


\section{Conclusion}

In this article we have shown that under realistic conditions when short sales are allowed, it is possible, and indeed quite easy, for a centralized decision maker to form an optimal overall portfolio while employing multiple outside portfolio managers ${ }^{16}$. This is on contrast to the assertions in the practitioner literature that argue this is not possible or possible only with full information. Outside managers should be willing to supply the information the CDM needs in our models since it does not require them to reveal private information on individual securities. Managers should be hesitant to reveal information on individual securities, since it is useful for multiple portfolios and to reveal it opens up the possibility of resale or direct use of the information.

When short sales are not allowed and if there is a single active manager to combine with passive indexes, a solution exists if it is optimum for the manager to place some funds in each index and/or the indexes (as opposed to the securities) can be sold short $^{17}$. When short sales are not allowed and there are multiple active managers, the previous analysis holds as long as a forecast of a negative alpha by a manager is taken to convey no information and the manager is simply told not to hold securities with negative alpha.

We have shown that in the case of multiple managers, if short sales are not allowed and the centralized manager makes use of estimates of negative alphas as well as positive alphas, a general optimum solution does not exist.

\footnotetext{
${ }^{16}$ Allowing short sales is an increasingly realistic case with the ability to use futures to short and with funds like hedge funds routinely shorting.

${ }^{17}$ The assumption that indexes can be sold short becomes increasingly realistic over time as exchange traded funds and futures have been created for an increasing number of indexes. It can be shown if the indexes cannot be sold short, a solution still exists as long as one and only one index is not held long
} 


\section{Bibliography}

Baks, Klaas, Andrew Metrick and Jessica Wachter (2001), "Should investors abort all actively managed mutual funds?" Journal of Finance, Vol. 56, pp 45-86.

Baway, Vijay, Stephen Brown and Roger Klein (1979), "Estimation risk and optimal portfolio choice," (North Holland, Amsterdam).

Di Bartolomeo (1999), “A radical proposal for the operation of multi-manager investment funds," Northfield Information Services.

Dypvig, Philip, Heber Farnsworth, and Jennifer Carpenter (2000), "Portfolio performance and agency," unpublished manuscript, New York University.

Elton, Edwin J., Martin J. Gruber and Manfred Padberg (1976). Simple criteria for optimal portfolio selection. Journal of Finance Vol. 11, pp 1341-1357.

Elton, Edwin J., Martin J. Gruber, and Padberg Manfred (1979), “Simple criteria for optimal portfolio selection," in Edwin J. Elton and Martin J. Gruber Portfolio Theory: Twenty-Five Years Later. Amsterdam, North Holland. 
Ferrell, James L. (1976), “The multi-index model and practical portfolio analysis," Research Foundation of the Institute of ? Financial Analysts.

Grinold, Richard, and Ronal Kaken (1965), Active Portfolio Management, Probus Publishing, Chicago, Illinois.

Kihlstrom, R. E. (1988), “Optimal contracts for security analysts and portfolio managers," Studies in Banking and Finance, 5, 291-325.

Lintner, John (1965), “The valuation of risk assets on the selection of investments in stock portfolios in capital budgets," Review of Economics and Statistics 47, 13-37.

Longtreth, Bevis (1986), Modern Investment Management and the Prudent Man Rule. Oxford University Press.

Marhsak, J. and Radner, R. (1972), “Economic theory of teams,” New Haven, CT. Yale University Press.

New York State Estates Powers and Trust Law. Section 11-2.3, Subsection (b) (4) (c).

Ohlson, Jim (1979), "Residual (API) analysis and the private value of information," Journal of Accounting Research, Vol 17, 2, Autumn, 506-527. 
Ohlson, Jim (1975), "The complete ordering of information alternatives for a class of Portfolio selection models," Journal of Accounting Research, Autumn.

Pastor, Lubos, and Robert Stambaugh (2001), "Investing in equity mutual funds." Unpublished manuscript, Wharton School of Business.

Radner, Roy (1962), “Team decision problems,” Annals of Mathematical Statistics March.

Rosenberg, Barr (1997), "Institutional investment with multiple portfolio managers," Proceedings of the Seminar on the Analysis of Security Prices, University of Chicago, $55-160$.

Sharpe, W.F. (1981). Decentralized investment management. Journal of Finance Vol. 36, pp. 217-234.

Stoughton, N. M. (1993), "Moral hazard and the portfolio management problem," Journal of Finance, 48(5), 2009-2028.

Treynor, Jack and Fisher Black (1973). How to use security analysis to improve portfolio selection. Journal of Business 46, 66-86. 
Zender, J. F. (1988), “Asymmetric Information in Financial Markets,” Ph.D. thesis, Yale University. 